\title{
The spatial extent of polycyclic aromatic hydrocarbons emission in the Herbig star HD 179218
}

\author{
A. S. Taha ${ }^{1,2}$, L. Labadie ${ }^{1}$, E. Pantin ${ }^{3}$, A. Matter ${ }^{4}$, C. Alvarez ${ }^{5}$, P. Esquej ${ }^{6}$, R. Grellmann ${ }^{1}$, \\ R. Rebolo ${ }^{7}$, C. Telesco ${ }^{8}$, and S. Wolf ${ }^{9}$ \\ ${ }^{1}$ I. Physikalisches Institut, Universität zu Köln, Zülpicher Straße 77, 50937 Köln, Germany \\ e-mail: maotahni@ph1.uni-koeln.de, labadie@ph1.uni-koeln.de \\ 2 Department of Astronomy, Faculty of Science, University of Baghdad, Baghdad - Aljadirya, Iraq \\ ${ }^{3}$ Laboratoire AIM, CEA/DRF-CNRS-Université Paris Diderot, IRFU/DAS, 91191 Gif-sur-Yvette, France \\ ${ }^{4}$ Laboratoire Lagrange, CNRS UMR 7293, UNS - Observatoire de la Côte d'Azur, BP 4229, 06304 Nice Cedex 4, France \\ ${ }^{5}$ W. M. Keck Observatory, 65-1120 Mamalahoa Hwy, Kamuela, HI 96743, USA \\ ${ }^{6}$ European Space Astronomy Centre (ESAC)/ESA, P.O. Box 78, 28690 Villanueva de la Cañada, Madrid, Spain \\ ${ }^{7}$ Instituto de Astrofisica de Canarias, C/ Via Lactea s/n, La Laguna, 38200 Tenerife, Spain \\ ${ }^{8}$ Department of Astronomy, University of Florida, Gainesville, FL 32611, USA \\ ${ }^{9}$ Institute for Theoretical Physics and Astrophysics, University of Kiel, Leibnizstr. 15, 24118 Kiel, Germany
}

Received 27 September 2017 / Accepted 9 November 2017

\begin{abstract}
Aims. We investigate, in the mid-infrared, the spatial properties of the polycyclic aromatic hydrocarbons (PAHs) emission in the disk of HD 179218, an intermediate-mass Herbig star at $\sim 300 \mathrm{pc}$.

Methods. We obtained mid-infrared images in the PAH-1, PAH-2 and Si-6 filters centered at 8.6, 11.3, and $12.5 \mu \mathrm{m}$, and $N$-band low-resolution spectra using CanariCam on the 10-m Gran Telescopio Canarias (GTC). We compared the point spread function (PSF) profiles measured in the PAH filters to the profile derived in the Si-6 filter, where the thermal continuum emission dominates. We performed radiative transfer modeling of the spectral energy distribution (SED) and produced synthetic images in the three filters to investigate different spatial scenarios.

Results. Our data show that the disk emission is spatially resolved in the PAH-1 and PAH-2 filters, while unresolved in the Si-6 filter. Thanks to very good observing conditions, an average full width at half maximum (FWHM) of $0.232^{\prime \prime}, 0.280^{\prime \prime}$ and $0.293^{\prime \prime}$ is measured in the three filters, respectively. Gaussian disk fitting and quadratic subtraction of the science and calibrator PSFs suggests a lower-limit characteristic angular diameter of the emission of $\sim 100$ mas, or $\sim 30$ au. The photometric and spectroscopic results are compatible with previous findings. Our radiative transfer (RT) modeling of the continuum suggests that the resolved emission should result from PAH molecules on the disk atmosphere being UV-excited by the central star. Simple geometrical models of the PAH component compared to the underlying continuum point to a PAH emission uniformly extended out to the physical limits of the disk model. Furthermore, our RT best model of the continuum requires a negative exponent of the surface density power-law, in contrast with earlier modeling pointing to a positive exponent.

Conclusions. We have spatially resolved - for the first time to our knowledge - the PAHs emission in the disk of HD 179218 and set constraints on its spatial extent. Based on spatial and spectroscopic considerations as well as on qualitative comparison with IRS 48 and HD 97048, we favor a scenario in which PAHs extend out to large radii across the flared disk surface and are at the same time predominantly in an ionized charge state due to the strong UV radiation field of the $180 L_{\odot}$ central star.
\end{abstract}

Key words. protoplanetary disks - radiation mechanisms: non-thermal - stars: variables: T Tauri, Herbig Ae/Be

\section{Introduction}

Circumstellar disks around pre-main sequence stars constitute the reservoir of gas and dust out of which planetary systems may form. The study of their morphological structure and spectroscopic content, as well as of their temporal evolution provides important information used to constrain the models of planet formation. From the spectral shape of the infrared excess, Meeus et al. (2001) classify intermediate-mass Herbig stars as two groups based on the possible geometry of their dust disks. Group I objects exhibit a flared disk geometry while group II sources correspond to a flatter geometry of the circumstellar disk. Recently, a number of high-angular-resolution and high-sensitivity spectroscopic studies have provided evidence showing the complex spatial structure of disks in the form of (pre-) transitional or "gaped" disks eventually harboring spiral structures (Calvet et al. 2002; Furlan et al. 2006; Espaillat et al. 2010; Gräfe et al. 2011; Tatulli et al. 2011; Benisty et al. 2015). A powerful tracer of the possible flared structure of the disk is found in the polycyclic aromatic hydrocarbons (PAHs) midinfrared emission bands found in a significant number of Herbig stars (Acke et al. 2010). When in the direct line-of-sight of the central star, PAH molecules on the surface of a flared disk can be electronically UV-excited by stellar photons even at large distances in the disk and cool down by re-emitting in the $\mathrm{CH}$ - or CC- stretching and bending modes at characteristic wavelengths (e.g., at $6.3,8.6$, or $11.3 \mu \mathrm{m}$ ). High-spatial-resolution imaging and long-slit spectroscopy in the PAH bands has exploited these properties to investigate the outer disk structure in HD 97048 (Lagage et al. 2006), or to trace possible gas flows through the 
disk's gaps (Maaskant et al. 2014). PAHs emission also trace the presence of very small grains mixed with the gas at high elevation above the midplane and have a significant influence on the structure of the disk by contributing to the gas heating (Habart et al. 2004).

In this paper we present CanariCam (Telesco et al. 2003) high-angular-resolution mid-infrared imaging and spectroscopy data for HD 179218, a Herbig star located at $290 \mathrm{pc}^{1}$ with a B9 spectral type that harbors a circumstellar disk primarily revealed through its infrared excess. Meeus et al. (2001) classified HD 179218 as a group-Ia source, which suggests a flared disk structure. A large amount of crystalline grains are found in this source, which points to significant dust processing (Bouwman et al. 2001). The latter two papers report the detection of PAHs at $8.6 \mu \mathrm{m}$ and at $11.3 \mu \mathrm{m}$. These detections were later confirmed and quantified by Juhász et al. (2010). Regarding the spatial structure of HD 179218's disk, Fedele et al. (2008) used MIDI/VLTI mid-infrared interferometry to show that HD 179218 could be a pre-transitional disk. Furthermore, the authors noticed a lower visibility shortward of $9 \mu \mathrm{m}$ that may result from a larger size scale of the PAH emission with respect to the continuum, but the scenario remains speculative based on the quality of the MIDI data.

Here, we aim at resolving the disk emission in two PAH bands in order to constrain the global structure of the disk on the large scale (Wolf et al. 2012) as we may assume that the PAH molecules remain co-spatial with the gas (Woitke et al. 2016). The paper is structured as follows: Sect. 2 summarizes the new observations conducted on the GTC. Section 3 presents the observational imaging and spectroscopic results and Sect. 4 focuses on the derivation of the emission characteristic size. Section 5 presents our modeling to investigate the origin of the resolved emission, while our results are discussed in Sect. 6.

\section{Observations and data reduction}

We used CanariCam (Telesco et al. 2003), the mid-infrared (7.5-25 $\mu \mathrm{m})$ imager with spectroscopic capabilities of the Gran Telescopio canarias in La Palma, Spain. Although the GTC has an equivalent $10.4-\mathrm{m}$ primary mirror, the diffraction limit achievable with CanariCam is set by a cold circular pupil stop equivalent to 9.4-m used to optimize the sensitivity. CanariCam holds a Raytheon $320 \times 240 \mathrm{Si}$ :As detector which covers a field of view of $26^{\prime \prime} \times 19^{\prime \prime}$. The detector plate scale is $0.08^{\prime \prime} / \mathrm{pixel}^{2}$.

Images were taken in the PAH-1 $(8.6 \mu \mathrm{m}, \Delta \lambda \approx 0.43 \mu \mathrm{m})$, PAH-2 $(11.3 \mu \mathrm{m}, \Delta \lambda \approx 0.60 \mu \mathrm{m})$ and Si-6 $(12.5 \mu \mathrm{m}, \Delta \lambda \approx$ $0.7 \mu \mathrm{m})$ filters. A standard chop-nod procedure with $8^{\prime \prime}$ throw was used. Chopping is hence performed within the detector, which means that one central positive image of the source flanked by two negative images is obtained after data reduction.

The observations of HD 179218 have been obtained on June 8, 2015, and September 15, 2015. The observing log is detailed in Table 1. The observation sequence consisted in the observation of a point spread function (PSF) reference star, followed by the science object, followed again by a second and different PSF reference star. The positions in the sky of the calibrators were chosen to minimize the difference in the parallactic angles at the time of observation. This ensures a similar image quality for the science and calibration targets. We have performed this technique of observation for all three filters mentioned above.

\footnotetext{
1 New Gaia parallax, see Sect. 5.1.

2 Cf. http://www.gtc.iac.es/instruments/canaricam/ canaricam.php
}

The average optical seeing extracted from the DIMM measurements was excellent during the June run, with values raging from $0.42^{\prime \prime}$ to $0.6^{\prime \prime}$. For the September run, the first calibrator suffered from poorer optical seeing $\left(\gtrsim 1^{\prime \prime}\right)$ in comparison to the following science target and second calibrator, which were observed in much better seeing conditions. The precipitable water vapor was measured between $7 \mathrm{~mm}$ and $8 \mathrm{~mm}$, which is suitable for good-quality observations in the $\mathrm{N}$ band. Low-resolution spectroscopic observations were obtained on July 1, 2015, under good weather conditions with $\sim 0.8^{\prime \prime}$ seeing and $10 \mathrm{~mm}$ precipitable water vapor. The spectroscopic calibration was obtained by observing two different Cohen standard stars before and after the science target. The $0.36^{\prime \prime}$ slit was used with a $6^{\prime \prime}$ throw.

The IDL pipeline iDealCam (Li 2014), which was customdesigned to reduce imaging data of CanariCam, was used for the data reduction. After removal of the thermal background, the pipeline produces individual frames (or saveset) of $\sim 2 \mathrm{~s}$ duration that can be combined in a long integration sequence. Savesets can be realigned along their centroid through two-dimensional Lorentzian $^{3}$ fit of the PSF prior to the final shift-and-add stacking. This allows reducing centering and tip-tilt errors that may otherwise lead to unwanted broadening of the PSF. Despite observing in the mid-infrared range with a good $\sim 0.5-0.6^{\prime \prime}$ average seeing, the atmospheric turbulence above a $10-\mathrm{m}$ class telescope still degrades the image quality, resulting in a nonfully diffraction-limited PSF. Depending on the instantaneous strength of turbulence, the image quality of individual savesets can be significantly affected, which appears in the form of distorted and elongated PSFs. For all our targets, we visually inspected each saveset of the CanariCam dataset and discarded the most corrupted images, that is, where the PSF clearly departs from circular shape. We note that in order to avoid biases by selecting only the "best" images, we applied the same visual criterion for frame selection to both the PSF reference and the science targets. The saveset duration of $\sim 2 \mathrm{~s}$ is the same for the science and reference targets, which also have similar fluxes in the $\mathrm{N}$ band. The resulting on-source integration time for the final stacked images is reported in Table 2 together with the percentage of frame selection. The final stacked image is obtained after re-centering and co-addition of the "positive" and "negative" images resulting from the data reduction (see Sect. 3.2). Except for the first calibrator in the Si- 6 filter for which the seeing conditions were not sufficiently good (see later), the procedure of frame selection resulted in discarding about $20 \%$ of the frames. We inspected approximately 448 savesets for the science target and approximatively 96 savesets for both reference stars and for the three filters. The frame selection resulted in an effective on-source total integration time for HD 178219 target of 1572 s, 1653 s and 1667 s for, respectively, the PAH-1 PAH-2 and Si-6 filters. Figure 1 illustrates the result of the process of frame selection on the full width at half maximum (FWHM) distributions used in our analysis. The FWHM is estimated by fitting of a Lorentzian function. Points have been removed when they correspond to a visually distorted PSF. Some points lie below the theoretical diffraction limit of the telescope (dashed line) due to improper Lorentzian fit of the corresponding PSF and have been consequently removed for both the science and PSF calibrators. Importantly, we note that comparatively large values of the FWHM are not removed from the distributions as long as the visual inspection of the corresponding saveset is compatible with a circular PSF. In this way, we aim at limiting bias effects in the analysis of the FWHM distributions.

The FITPSF procedure from F. Varosi's IDL library was used. 
Table 1. Observing log.

\begin{tabular}{ccccccccc}
\hline \hline Object & Start [UT] & End [UT] & Filter & On source [s] & PWV [mm] & Airmass & Seeing ["] & Flux standard \\
\hline HD 169414 & $03: 35$ & $03: 46$ & PAH-1 & 397 & 7.8 & 1.04 & 0.60 & PSF standard star \\
HD 179218 & $04: 01$ & $04: 49$ & PAH-1 & 1853 & 8.1 & 1.06 & $0.42 \pm 0.03$ & Science \\
HD 187642 & $05: 37$ & $05: 48$ & PAH-1 & 397 & 7.6 & 1.17 & 0.55 & PSF standard star \\
HD 169414 & $03: 47$ & $03: 57$ & PAH-2 & 417 & 7.7 & 1.06 & 0.51 & PSF standard star \\
HD 179218 & $04: 51$ & $05: 33$ & PAH-2 & 1807 & 8.1 & 1.14 & $0.45 \pm 0.02$ & Science \\
HD 187642 & $05: 48$ & $05: 58$ & PAH-2 & 417 & 7.0 & 1.20 & 0.58 & PSF standard star \\
HD 169414 & $20: 55$ & $21: 06$ & Si-6 & 397 & 8 & 1.03 & 1 & PSF standard star \\
HD 179218 & $21: 40$ & $22: 28$ & Si-6 & 1853 & 7.7 & 1.08 & $0.60 \pm 0.03$ & Science \\
HD 187642 & $22: 34$ & $22: 44$ & Si-6 & 397 & 8.2 & 1.11 & 0.5 & PSF standard star \\
HD 169414 & $00: 38$ & $00: 49$ & LR Spec. & 176 & 11.3 & 1.0 & 0.85 & Telluric standard \\
HD 179218 & $01: 13$ & $02: 11$ & LR Spec. & 943 & 11.1 & 1.02 & 0.77 & Science \\
HD 187642 & $02: 32$ & $02: 43$ & LR Spec. & 176 & 10 & 1.06 & 0.80 & Telluric standard \\
\hline
\end{tabular}

Notes. Frames in the PAH-1 and PAH-2 filters were acquired on June 8, 2015. Frames in the Si-6 filter were acquired on September 15, 2015.
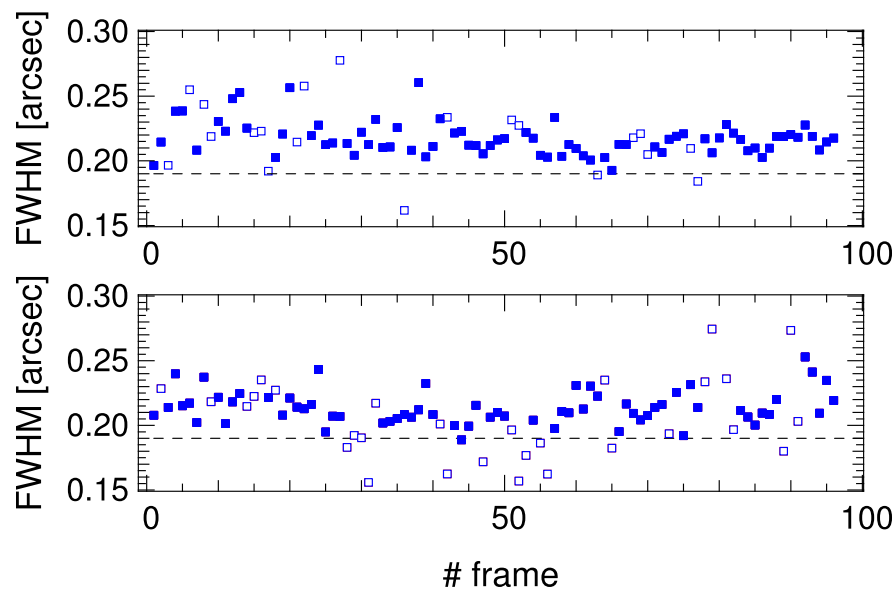

Fig. 1. Illustration of the frame selection on the PSF calibrators HD 187642 (top) and HD 169414 (bottom) for the PAH-1 filter. The full distribution is given by the filled + empty symbols. The filled squares correspond to the frames finally selected. The dashed line is the theoretical diffraction limit of the telescope at $8.6 \mu \mathrm{m}$. The FWHM is estimated by fitting of a Lorentzian function.

\section{Observational results}

\subsection{Statistics of the full width at half maximum}

Similarly to Moerchen et al. (2007), we have explored the statistical behavior of the PSF FWHM distribution obtained after Lorentzian fitting of the individual selected savesets. By treating statistically individual 2 s-short images and employing sub-pixel recentering for both the science and calibration targets, we minimize the influence of long-term biases (e.g., guiding errors, pupil rotation, and seeing fluctuations) that may result in the broadening of the final PSF when simply stacking the long sequence images. We extracted from the distribution of the FWHM data the mean and the error on the mean $\sigma / \sqrt{N}$, where $\sigma$ is the distribution standard deviation and $N$ is the number of savesets, respectively nodsets, in the distribution. Figure 2 shows the distribution of FWHM values of the individual savesets for each sequence calibrator-science-calibrator in the three filters. It is already possible to visually discriminate the vertical positioning of the bulk of the distribution for the science (blue open circles) and the adjacent calibrators (red open squares). The plots provide evidence that, for the PAH filters at $8.6 \mu \mathrm{m}$ and $11.3 \mu \mathrm{m}$, the

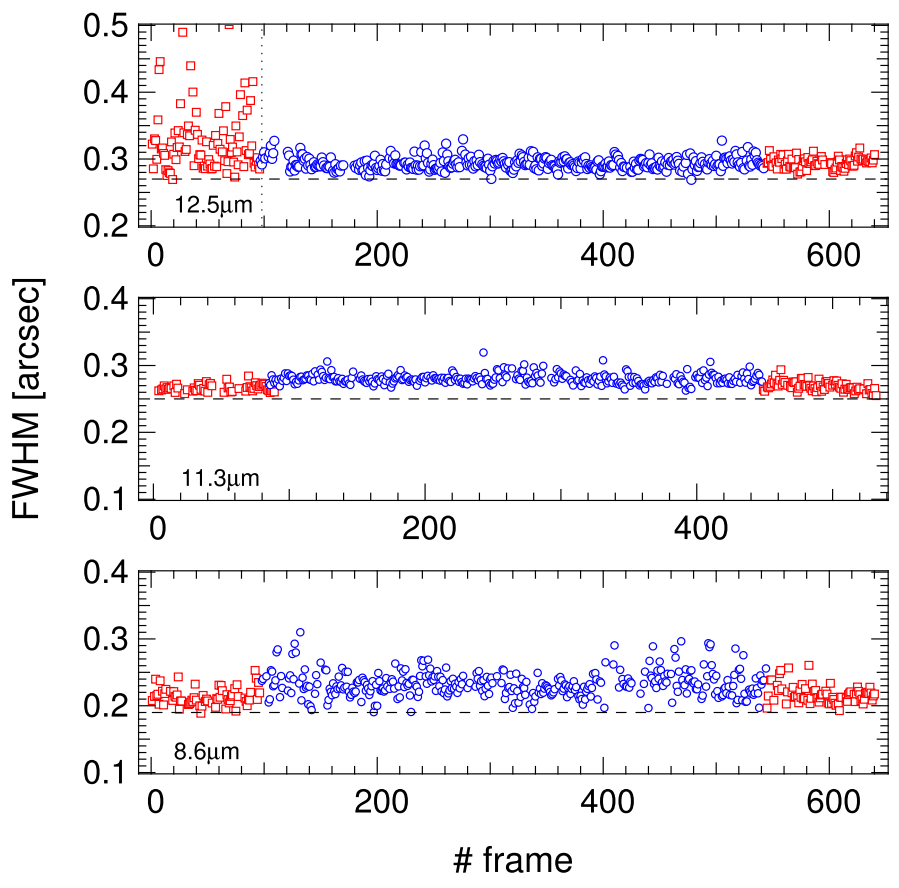

Fig. 2. Distribution as a function of time of the measured FWHM for the individual savesets. The blue open circles correspond to the science target HD 179218 and the red open squares correspond to the adjacent calibrators HD 169414 and HD 187642. The plots correspond, respectively from bottom to up, to the filters PAH-1, PAH-2 and Si-6. The integration time for the individual saveset for both the science and the calibrator is $2.1 \mathrm{~s}$ (PAH-1), $2.5 \mathrm{~s}$ (PAH-2) and $2.1 \mathrm{~s}$ (Si-6). The horizontal dashed lines show the theoretical diffraction limit of the GTC, which is $0.19^{\prime \prime}, 0.25^{\prime \prime}$ and $0.27^{\prime \prime}$ at respectively $8.6 \mu \mathrm{m}, 11.3 \mu \mathrm{m}$ and $12.5 \mu \mathrm{m}$, including the central obscuration. For the Si- 6 filter, the vertical dotted line around frame \#100 shows the effect of poor seeing on the measured FWHM distribution for the first calibrator.

FWHM of HD 179218 is on average larger that the FWHM of the adjacent calibrators. For the filter Si-6 at $12.5 \mu \mathrm{m}$, if we neglect the FWHM distribution of the first calibrator corrupted by poor seeing, the FWHM distribution for HD 179218 does not exhibit any remarkable deviation from that of the calibrator.

In Table 2 we report for the saveset distribution the estimated mean FWHM along with the corresponding $3 \sigma$ error for the science and the calibrators in the three different filters. For consistency, we compared the FWHM statistics obtained with 
Table 2. Measured mean FWHM and its resulting $3 \sigma$ uncertainty from the distribution of savesets.

\begin{tabular}{crcrr}
\hline \hline Object & On source [s] & $\begin{array}{c}\text { \% of frame } \\
\text { selection }\end{array}$ & FWHM $_{\mathrm{L}}\left[^{\prime \prime}\right]$ & FWHM $\left._{\mathrm{P}}{ }^{\prime \prime}{ }^{\prime \prime}\right]$ \\
\hline HD 169414 & 273 (PAH-1) & 69 & $0.213 \pm 0.004$ & $0.233 \pm 0.003$ \\
HD 179218 & 1501 (PAH-1) & 81 & $0.232 \pm 0.003$ & $0.255 \pm 0.002$ \\
HD 187642 & 314 (PAH-1) & 79 & $0.217 \pm 0.004$ & $0.234 \pm 0.003$ \\
HD 169414 & 287 (PAH-2) & 70 & $0.267 \pm 0.002$ & $0.282 \pm 0.002$ \\
HD 179218 & 1651 (PAH-2) & 91 & $0.280 \pm 0.001$ & $0.298 \pm 0.001$ \\
HD 187642 & 382 (PAH-2) & 91 & $0.268 \pm 0.002$ & $0.285 \pm 0.002$ \\
HD 169414 & $397($ Si-6) & - & - & - \\
HD 179218 & $1667($ Si-6) & 90 & $0.293 \pm 0.001$ & $0.313 \pm 0.002$ \\
HD 187642 & $364($ Si-6) & 91 & $0.294 \pm 0.002$ & $0.311 \pm 0.003$ \\
\hline
\end{tabular}

Notes. FWHM $_{\mathrm{L}}$ is estimated through a Lorentzian fit of the saveset's PSF. The term $\sigma$ is here the error on the mean of the distribution, that is, the estimated root mean square divided by $\sqrt{N}$ with $N$ being the number of selected frames. FWHM $\mathrm{P}_{\mathrm{P}}$ is estimated graphically from the PSF profile according to the definition of the FWHM. The reported $3 \sigma$ uncertainty is computed from the mean and error on the mean of the distribution of radial profile computed for each saveset. Therefore, the uncertainty obtained with both methods are comparable.

Table 3. Resolution criterion for HD 179218 compared to the adjacent calibrators following Moerchen et al. (2010).

\begin{tabular}{lccc}
\hline \hline Filter & FWHM $_{\text {sci }}-\mathrm{FWHM}_{\text {cal }}$ & $3 \sigma_{\text {tot }}$ & Resolved \\
\hline PAH-1 & $0.019 / 0.022($ Cal 1) & $0.005 / 0.004$ & Y \\
PAH-1 & $0.015 / 0.021($ Cal 2) & $0.005 / 0.004$ & Y \\
PAH-2 & $0.013 / 0.016($ Cal 1) & $0.002 / 0.002$ & Y \\
PAH-2 & $0.012 / 0.013($ Cal 2) & $0.002 / 0.002$ & Y \\
Si-6 & $0.001 / 0.002($ Cal 2) & $0.002 / 0.004$ & $\mathrm{~N}$ \\
\hline
\end{tabular}

Notes. For Cols. (2) and (3), the first term corresponds to the Lorentzian fit method and the second term corresponds to the direct FWHM graphical read from the PSF profile.

Lorentzian fitting to the statistics obtained by the direct graphical reading of the FWHM according to its definition. We can see that the Lorentzian fit approach systematically gives an average FWHM lower by $\leq 10 \%$ than the FWHM derived from a graphical reading. However, the relative trends between science and calibrators are reproducible. We observe that within the $99.7 \%$ confidence level the measured FWHM of the science target is larger than the one of the adjacent calibrators for both PAH filters, while it is the same in the Si-6 filter at $12.5 \mu \mathrm{m}$. We further assessed the resolved nature of HD 179218's emission by applying the criterion of Moerchen et al. (2010). Namely we compare the difference in FWHM between the science target and the calibrator star, that is,

$\mathrm{FWHM}_{\mathrm{sci}}-\mathrm{FWHM}_{\mathrm{cal}}$,

to the combined standard deviation (i.e., error) of the mean

$\sigma_{\mathrm{tot}}=\sqrt{\sigma_{\mathrm{cal}}^{2}+\sigma_{\mathrm{sci}}^{2}}$

With $\mathrm{FWHM}_{\mathrm{sci}}-\mathrm{FWHM}_{\mathrm{cal}} \geq 3 \sigma_{\text {tot }}$ the science source can be considered as spatially resolved. According to this criterion, we give in Table 3 the result of this analysis and show that the circumstellar emission around HD 179218 is resolved at $\geq 3 \sigma$ confidence level in the two PAH filters, but is unresolved at $12.5 \mu \mathrm{m}$.

\subsection{Retrieval of the PSF profile}

The final images are obtained after re-centering and stacking the individual savesets. Image re-centering has to be performed carefully to avoid a significant increase of the final FWHM typically observed in long observing sequences. A simple recentering step based on matching the individual image centroid induces an increase in the FWHM of the final stacked image by $\sim 15 \%$ compared to a single saveset. To improve the centering step, we realigned each saveset by minimizing the quadratic difference between the image of each normalized PSF and the image of the first PSF of the sequence taken as a reference. This operation is implemented with a sampling accuracy of the image of one fifth of a pixel. Such an approach led to a sharper PSF. Figure A.1 gives the Lorentzian-fitted FWHM of the stacked image as a function of the number of coadded savesets. The plots shows, within the limits of the available frames, that the FWHM of the stacked image tends to the statistical value found in Table 2.

After recentering the individual savesets as described above, the radial profiles for the calibrator and science targets is built for the different available filters. To construct such a profile along with its associated error bars, we extracted the profile for each individual recentered saveset and computed the mean and the error on the mean for each radial pixel. The results for the three different filters are shown in Fig. 5 for the science and calibrator PSF.

\subsection{Photometry}

We performed aperture photometric calibration of HD 179218 using HD 169414 and HD 187642 as Cohen photometric standards. With the two reference stars observed before and after the science target, we can also probe the long term photometric stability of the night. The aperture radius was optimized to $4 \times$ FWHM, or $\sim 1^{\prime \prime}$. This size is close to the standard $5 \times$ FWHM recommended by photometry manuals. The residual background was estimated in a surrounding ring with an inner and outer radius of $\sim 1.6^{\prime \prime}$ (20 pixels) and $\sim 2.4^{\prime \prime}$ (30 pixels), respectively. For each science and calibrator target the photometric accuracy is derived from the measurement of the standard deviation of the flux over the savesets time series. The values for the photometric standards in the CanariCam filters are taken from www.astro.ufl.edu/ dli/web/IDEALCAM_ files/iDealCam_v2.0.zip. The results of the photometric 
Table 4. Photometric calibration of HD 179218 in the CanariCam filters using the photometric standards reported in Col. (3).

\begin{tabular}{lccc}
\hline \hline Filter & Flux sci [Jy] & Cal & Flux cal [Jy] \\
\hline PAH-1 & $14.9 \pm 0.4$ & HD 169414 & 19.2 \\
PAH-1 & $15.3 \pm 0.4$ & HD 187642 & 43.3 \\
PAH-2 & $22.4 \pm 0.6$ & HD 169414 & 12.2 \\
PAH-2 & $22.1 \pm 0.4$ & HD 187642 & 25.4 \\
Si-6 & $16.8 \pm 1.7$ & HD 169414 & 9.9 \\
Si-6 & $16.2 \pm 0.7$ & HD 187642 & 20.8 \\
\hline
\end{tabular}

Notes. The reported errors are $3 \sigma$ uncertainties. The larger uncertainty obtained with HD 169414 in the Si-6 filters is due to the initial poorer conditions of the night. We note that the uncertainties reflect only the photometric stability of our measurement. It is however known that many of the Cohen standards show some variability, which results in an absolute photometric accuracy of about $10 \%$.

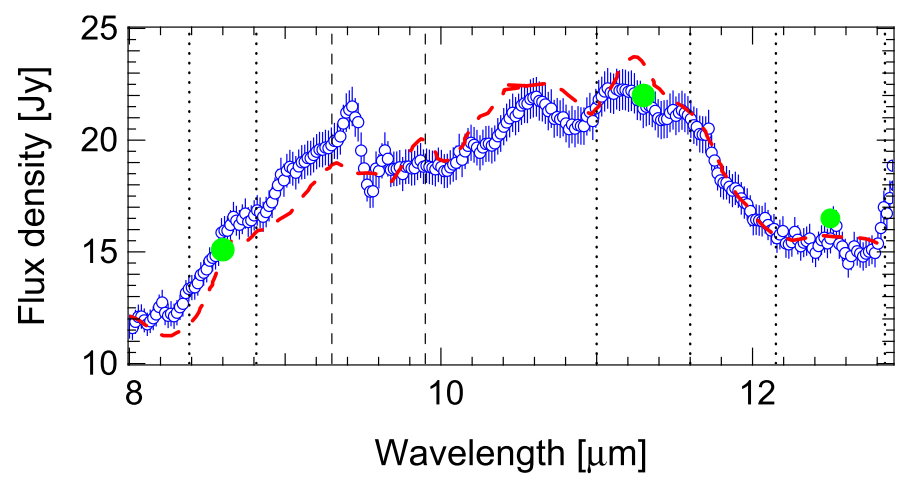

Fig. 3. Low-resolution spectrum of HD 179218 (open circles with error bars) scaled to the measured photometry at $11.3 \mu \mathrm{m}$ (green filled circles). The red dashed line is the corresponding Spitzer/IRS spectrum. The vertical dotted lines denote the bandwidth of the PAH-1, PAH-2, and $\mathrm{Si}$ 6 filters. The vertical dashed line indicates the region of the atmospheric ozone feature where proper spectroscopic calibration is cumbersome.

calibration are shown in Table 4 and are consistent with Spitzer spectroscopy data by Fedele et al. (2008) and Juhász et al. (2010).

\subsection{Spectroscopy}

The spectrum of HD 179218 was reduced with the RedCan pipeline (González-Martín et al. 2013) and is shown in Fig. 3. The reduced spectrum is found to overestimate the flux by $40 \%$ in comparison to our photometric values, which may have different causes (e.g., presence of cirrus or imperfect background subtraction). As we wish to make a relative comparison of the shape of the CanariCam spectrum and the Spitzer spectrum, the former one has been rescaled to the measured photometric values reported in Table 4. After rescaling, the relative comparison with the Spitzer spectrum shows good agreement in the shape with the visible peaks at $\sim 8.6-8.7 \mu \mathrm{m}, 10.6 \mu \mathrm{m}$, and $\sim 11.2-11.3 \mu \mathrm{m}$. Qualitatively, the flux density is slightly larger in the PAH-1 filter for CanariCam than for Spitzer, while it is lower in the PAH-2 filter. The spectral calibration in the 9.3-9.9 $\mu \mathrm{m}$ region is strongly affected by the ozone atmospheric feature and residuals of the data reduction can be seen. We also remark that the flux measured by CanariCam between 9 and $9.2 \mu$ m appears overestimated by 10 to $20 \%$ in comparison to ISO and Spitzer.
Table 5. Angular diameter of the circumstellar emission measured in the three different filters.

\begin{tabular}{lccc}
\hline \hline Calibrator & Filter & $D_{\mathrm{d}, \mathrm{L}}\left[{ }^{\prime \prime}\right]$ & $D_{\mathrm{d}, \mathrm{PSF}}\left[{ }^{\prime \prime}\right]$ \\
\hline HD 169414 & PAH-1 & $0.092 \pm 0.004$ & $0.103 \pm 0.003$ \\
HD 187642 & PAH-1 & $0.082 \pm 0.005$ & $0.101 \pm 0.003$ \\
HD 169414 & PAH-2 & $0.084 \pm 0.003$ & $0.096 \pm 0.002$ \\
HD 187642 & PAH-2 & $0.081 \pm 0.002$ & $0.087 \pm 0.002$ \\
HD 187642 & Si-6 & $\leq 0.024 \pm 0.009$ & $\leq 0.035 \pm 0.011$ \\
\hline
\end{tabular}

Notes. The uncertainty refers to the $1 \sigma_{\mathrm{d}}$ error as derived in Mariñas et al. (2011). The source is considered resolved if the deconvolved (in the sense of quadratic subtraction) diameter is larger than $3 \sigma_{\mathrm{d}}$. The subscript $\mathrm{L}$ and $\mathrm{P}$ refer to the Lorentzian fit and to a direct measurement of the PSF profile, respectively.

\section{Characteristic size of the emission}

\subsection{Quadratic subtraction of FWHMs}

For small differences in FWHM measurements, as in our case, PSF deconvolution is a delicate technique which strongly depends on assumptions made on the PSF and number of iterations. Similarly to Moerchen et al. (2010); Mariñas et al. (2011), an alternative to deconvolution is the estimate of the disk diameter from the quadratic subtraction of the science PSF and calibrator PSF derived in Sect. 3 through $D_{\mathrm{d}}^{2}=\mathrm{FWHM}_{\mathrm{sci}}^{2}-\mathrm{FWHM}_{\mathrm{cal}}^{2}$, where $D_{\mathrm{d}}$ is the estimated disk diameter. The errors associated to $D_{\mathrm{d}}$ are calculated following Eq.(2) in Mariñas et al. (2011). Our estimates are reported in Table 5. Assuming a distance of $293 \mathrm{pc}$ (cf. Sect. 5), we found in the two filters a comparable characteristic diameter of $\sim 24-30$ au. On average, the disk emission appears slightly more extended in the PAH-1 filter than in the PAH-2 filter. The disk emission is found to be unresolved in the Si- 6 filter in the sense of the $3 \sigma_{\mathrm{d}}$ criterion.

\subsection{Gaussian disk}

We complemented the previous estimate with a simple approach to determine the characteristic size of the resolved emission. Namely, we model the emission as a two-dimensional face-on Gaussian disk convolved with the telescope PSF in the corresponding filters. This model only depends on the FWHM of the Gaussian function, though it might not be always sophisticated enough to reproduce the full shape of the PSF profile (core+wings). The characteristic size is estimated by visually matching the synthetic profile to the science profile within the experimental error bars. In this analysis we used the final images after recentering and stacking.

In the PAH-1 filter, a Gaussian disk with a FWHM of $95 \pm 6$ mas reproduces our science PSF profile, whereas a Gaussian disk with a FWHM $\leq 22 \pm 7$ mas would remain spatially unresolved. In the PAH-2 filter, the Gaussian disk model fitting our science profile has a FWHM of $101 \pm 7$ mas, and the unresolved disk would have a FWHM $\leq 25 \pm 7$ mas. Finally, in the Si-6 filter the Gaussian disk must have a FWHM $\leq$ $36 \pm 7$ mas so as not to exceed the FWHM of the science profile. This analysis confirms that the disk emission in HD 179218 is resolved in the PAH filters and unresolved in the $12.5 \mu \mathrm{m} \mathrm{Si}-6$ filter. However, it is not possible to conclude within the error bars on a difference in the angular size of the emission in the PAH-1 and PAH-2 filters. 
Table 6. Stellar parameters assumed in this study.

\begin{tabular}{cccccc}
\hline \hline$L_{*}\left[L_{\odot}\right]$ & $M_{*}\left[M_{\odot}\right]$ & $T_{*}[K]$ & $R_{*}\left[R_{\odot}\right]$ & $\mathrm{A}_{V}[\mathrm{mag}]$ & $d[\mathrm{pc}]$ \\
\hline $180^{a}$ & $3.66^{a}$ & $9640^{a}$ & $4.8^{a}$ & $0.63^{b}$ & $293^{c}$ \\
\hline
\end{tabular}

Notes. ${ }^{(a)}$ extracted from Alecian et al. (2013); ${ }^{(b)}$ fit of the optical photometry; ${ }^{(c)}$ Gaia parallax.

\section{Modeling}

Our imaging results show that the circumstellar emission around HD 179218 is spatially resolved in the two PAH filters whereas it remains unresolved at $12.5 \mu \mathrm{m}$. We attempt to identify the origin of the extended emission. A natural comparison arises with the case of HD 96048 for which Lagage et al. (2006) resolve the emission of polycyclic aromatic hydrocarbons at the surface of the flared disk in direct view of the central star. In order to test this possible configuration in the case of HD 179218, we adopt the following strategy: we develop a radiative transfer model of a disk that simultaneously fits the spectral energy distribution (SED) and is spatially unresolved in the synthetic CanariCam image at $12.5 \mu \mathrm{m}$. The idea is to constrain the disk's size in the $12.5 \mu \mathrm{m}$ band, which is dominated by the dust thermal emission and shows no significant presence of PAH emission (Acke et al. 2010; Juhász et al. 2010). A PAH-free disk emission model with the same parameters is then produced at 8.6 and $11.3 \mu \mathrm{m}$ from which a synthetic observational profile can be extracted and compared to our observations. A similar strategy was successfully used by Honda et al. (2012) to model the gap's size of HD 169142 in the Q band. In this approach, the modeling of the thermal emission at $12.5 \mu \mathrm{m}$ gives us an upper limit on the outer disk's dimension while the inner regions are unresolved with CanariCam. If the 8.6 and $11.3 \mu \mathrm{m}$ synthetic profiles are spatially unresolved, this would be a good indication that the observed resolved emission is not of thermal origin (in the sense of "continuum" origin). On the contrary, in case the 8.6 and $11.3 \mu \mathrm{m}$ synthetic profiles are found to be spatially resolved, further observational constraint needs to be added through, for instance, mid-infrared interferometric data to be conclusive on the nature of the resolved emission in the PAH bands.

Dominik et al. (2003) proposed a first radiative transfer model based on a single disk geometry with an outer radius of $30 \mathrm{au}$ and a positive power-law index $p=2$ of the surface density. In a statistical study of HAeBe's disks, Menu et al. (2015) used simple geometrical temperature gradient models in combination with mid-infrared interferometry data to infer a half-light radius of the disk of $7 \pm 1.2$ au at $254 \mathrm{pc}$. Using nulling interferometry, Liu et al. (2007) derived a radius of $13.5 \pm 3$ au based on a ring-like disk model at a similar distance of $244 \mathrm{pc}$. Moreover, Fedele et al. (2008) applied an achromatic geometrical disk model to VLTI/ MIDI data and proposed as their best solution a two-component pre-transitional disk structure with an inner disk extending from $0.3-3$ au and an outer component whose bulk mid-IR emission lies in a 13-22 au region at 240 pc.

\subsection{Description of the disk model}

We developed radiative transfer disk models for HD 179218 aiming at constraining simultaneously the spectral energy distribution (SED) and the imaging data on the source. We used for this purpose the well-established Monte-Carlo code RADMC3D (Dullemond \& Dominik 2004) that permits one to synthesize disk images and SEDs. Assuming a disk in vertical hydrostatic equilibrium and perfect gas/dust coupling, the dust density in $\mathrm{g} . \mathrm{cm}^{-3}$ is modeled analytically according to

$\rho(r, z)=\frac{\Sigma(r)}{H(r) \sqrt{2 \pi}} \exp \left[-\frac{1}{2}\left(\frac{z}{H(r)}\right)^{2}\right]$

where $\rho(r, z)$ is described via the parametrized dust surface density $\Sigma(r)=\Sigma_{\text {out }}\left(r / r_{\text {out }}\right)^{p}$ and dust scale height $H(r)=$ $H_{\text {out }}\left(r / r_{\text {out }}\right)^{(1+\beta)}$, and $p$ is the surface density exponent and $\beta$ the disk flaring exponent. The subscript out refers to the outer radius of each disk component considered.

A difference with respect to earlier models is that we assume more recent estimates of the stellar parameters and parallax, which naturally influences the radiative transfer calculation and the production of the synthetic images. We used for the central star the parameters from Alecian et al. (2013), namely a luminosity $L_{*}=180 L_{\odot}$, a radius $R_{*}=4.8 R_{\odot}$, a mass $M_{*}=3.66 M_{\odot}$ and an effective temperature $T_{\text {eff }}=9640 \mathrm{k}$. A luminosity of $80-100 L_{\odot}$ was typically assumed in the earlier works. Based on a recent Gaia parallax measurement ${ }^{4}$ of $3.41 \pm 0.35$ mas, we assume a distance of $293 \mathrm{pc}$ rather than the $240 \mathrm{pc}$ found in the literature. The experimental SED of the system is taken from Acke \& van den Ancker (2004) and contains photometric data from the literature as well as spectroscopic data from ISO.

The RT grid extends from the dust sublimation radius to $200 \mathrm{au}$. The disk temperature distribution is computed through a first Monte Carlo run using $10^{6}$ photon packets. Since isotropic scattering is considered in our modeling, the scattering source function is then computed at each wavelength through an additional Monte Carlo run using $3 \times 10^{5}$ (resp. $5 \times 10^{4}$ ) photon packets for the images (resp. for the SED). A ray tracing method is then applied to compute the synthetic SED and images (at 8.6, 11.3, and $12.5 \mu \mathrm{m}$ ). In our RT modeling, we do not include PAHs, although they are clearly present, and model only the dust continuum. This is discussed later in the paper.

Following Fedele et al. (2008), we assume as a starting baseline a passive irradiated pre-transitional disk structure consisting of an inner narrow ring, a low dust density gap region and a larger outer disk. The outer component is decomposed into a warm disk atmosphere component that will dominate the midIR emission, and a colder mid-plane component optically thick at $10 \mu \mathrm{m}$ that will dominate the far-IR and sub-mm emission. The outer disk warm atmosphere will mostly influence our data in the mid-infrared (see Sects. 5.3 and 5.4).

\subsection{The dust opacities}

The continuum emission being entirely dominated by the dust, the grain composition and resulting opacity influences the shape and values of the SED from the near-IR to the sub-mm. As we do not aim at a detailed fit of the spectral feature already done elsewhere, our approach is to assume opacity laws detailed in the literature in order to place ourselves in a realistic case. For the outer disk, we assumed a composition of $90 \%$ amorphous silicate grains and $10 \%$ crystalline enstatite grains following the findings of Juhász et al. (2010). We assumed a size distribution $\propto a^{-3.5}$ (Mathis et al. 1977) from $0.1 \mu \mathrm{m}$ to $100 \mu \mathrm{m}$ for the amorphous grain population and a fixed size of $2 \mu \mathrm{m}$ for the enstatite grain population. The midplane is populated with larger amorphous silicate grains to reflect dust sedimentation, with sizes ranging from $10 \mu \mathrm{m}$ to $1 \mathrm{~mm}$ following a similar power-law size distribution to the one before. Finally, the inner

4 http://gea.esac.esa. int/archive/ 
Table 7. Best-model parameters resulting from the $\chi^{2}$ minimization.

\begin{tabular}{lcccccccc}
\hline \hline & $r_{\text {in }}[\mathrm{au}]$ & $r_{\text {out }}[\mathrm{au}]$ & $H_{\text {out }} / r_{\text {out }}$ & $M_{\text {dust }}\left[M_{\odot}\right]^{c}$ & $p$ & $\beta$ & $i\left[^{\circ}\right]^{d}$ & P.A. $\left[{ }^{\circ}\right]^{d}$ \\
\hline Inner Disk & $1.1^{a}$ & $3^{a}$ & $0.02^{a}$ & $1.46 \times 10^{-5^{a}}$ & $0^{a}$ & $1 / 7$ & 57 & 23 \\
Gap & $3.1^{a}$ & $9.9^{a}$ & $0.1^{a}$ & $1.83 \times 10^{-13^{a}}$ & $0^{a}$ & $1 / 7$ & 57 & 23 \\
Outer Disk & $10^{a+b}$ & $80^{a+b}$ & $0.12^{a}$ & $1.46 \times 10^{-4^{a}}$ & $-1.5^{a+b}$ & $1 / 7$ & 57 & 23 \\
\hline
\end{tabular}

Notes. ${ }^{(a)}$ The parameters fitted from the SED. ${ }^{(b)}$ The parameters fitted from the $12.5 \mu \mathrm{m}$ PSF profile. The parameters $\beta, i$ and P.A. are not fitted. ${ }^{(c)}$ The surface density $\Sigma_{\text {out }}$ in Eq. (3) is parametrized through the mass $M_{\text {dust }}$. A gas-to-dust ratio of 100 is assumed. ${ }^{(d)}$ From Fedele et al. (2008).

disk/gap is populated with a mixture of amorphous silicate and highly refractory carbon grains at a ratio of approximately $9: 1$, respectively, in agreement with Dominik et al. (2003).

\subsection{Fitting procedure: SED and $12.5 \mu \mathrm{m}$ image}

The procedure consists in best-fitting the SED through a $\chi^{2}$ minimization and verifying a posteriori that the corresponding synthetic image is unresolved at $12.5 \mu \mathrm{m}$. For a given radiative transfer model, we thus produced a synthetic SED to be compared to the observational SED, and a synthetic disk image at $12.5 \mu \mathrm{m}$ that we convolved with our PSF reference star in the Si-6 filter. A radial profile is then extracted to be compared with the observed profile at $12.5 \mu \mathrm{m}$.

As a first step, we run our radiative transfer code to identify a reference baseline model (RBM) that provides a good visual fit to the SED without PAH. This model assumes the properties aforementioned (central star, mineralogy) as well as a pre-transitional disk structure (inner disk + gap + outer disk; Fedele et al. 2008). A first exploration of the parameters shown in Table 7 (cf. caption) allows us to converge towards a possible solution for the RBM based on the SED fit. As a second step, on the basis of this RBM, we determined which parameters influence most significantly the mid-IR profiles and refine our search on these parameters by including the information on the $12.5 \mu \mathrm{m}$ PSF profile. In this way, we avoid varying all the parameters of the model to minimize degeneracy effects.

(a) Inner disk: the inner radius is fixed at $1.1 \mathrm{au}$, which roughly fits the dust sublimation radius given our stellar parameters. We then tested the influence of the outer radius of the inner disk by varying it out to $5 \mathrm{au}$ and varying the exponent of the power law from $p=-2$ to $p=2$. This corresponds to a range of exponents typically found for disk models (Dominik et al. 2003). We observe a mild influence of these parameters on the SED in the 2-3 $\mu$ m near-IR region and no impact on the PSF profiles at 8.6, 11.3, and in particular at $12.5 \mu \mathrm{m}$. The inner disk scale height and flaring index have also no measurable impact on the mid-infrared profiles.

(b) Gap: this low-density region hosts a dust mass of $\sim 10^{-13} M_{\odot}$ in the RBM. The power-law exponent of the surface density was varied from -2 to +2 . This parameter did not impact the various PSF profiles either.

(c) Outer disk: the inner and outer radii of the outer disk component, $R_{\mathrm{i}}$ and $R_{\mathrm{o}}$, along with the surface density power-law exponent were found to influence most significantly the PSF profiles at $12.5 \mu \mathrm{m}$ and the SED at mid-IR up to sub$\mathrm{mm}$ wavelengths. We therefore concentrated on these three parameters in what follows.

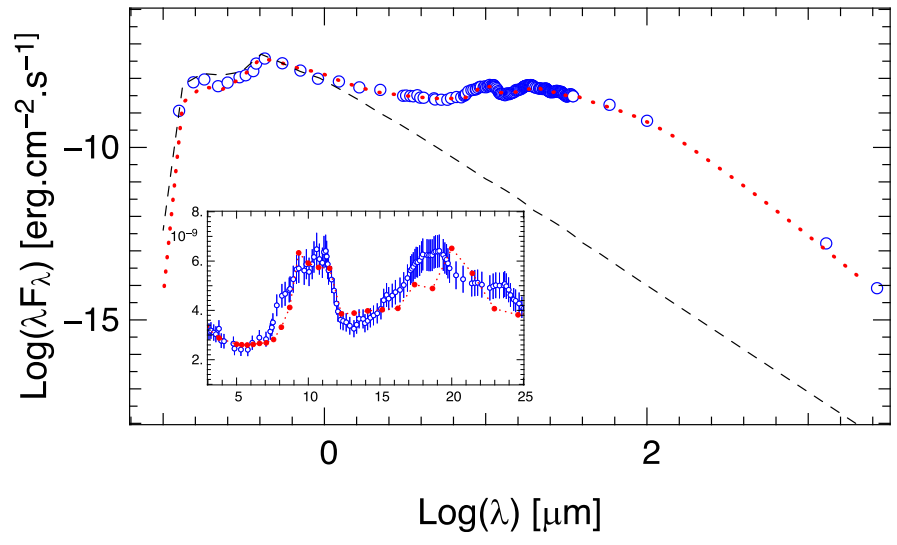

Fig. 4. Spectral energy distribution of HD 178219 (blue open circles) and overplotted synthetic SED from radiative transfer modeling (red dotted line) with a resulting $\chi_{r}^{2}=1.9$. The line-of-sight extinction is $A_{v}=0.63$ and the distance $d=293 \mathrm{pc}$. The photometry error bars are conservatively assumed to be $10 \%$. The inset shows a view on the $3-25 \mu \mathrm{m}$ region of the SED. The black dashed line is the stellar photosphere.

\subsection{Exploration of the outer disk's parameters $p, R_{\mathrm{i}}$, and $R_{\mathrm{o}}$}

We have conducted a small parameter search by varying the power law $p$ in the range $\{-2,+2\}$ in steps of 0.5 , the inner radius of the outer disk $R_{\mathrm{i}}$ in the range $\{8 \mathrm{au}, 12 \mathrm{au}\}$ and the outer radius of the outer disk $R_{\mathrm{o}}$ in the range $\{30 \mathrm{au}, 150 \mathrm{au}\}$. We have simultaneously compared our synthetic SED and $12.5 \mu \mathrm{m}$ PSF profile to our observations. Table B.1 gives the value of the non-reduced $\chi^{2}$ for the SED fit as a function of $\left(p, R_{\mathrm{i}}, R_{\mathrm{o}}\right)$ and highlights the models for which the PSF profile at $12.5 \mu \mathrm{m}$ is either spatially resolved or unresolved. The parameter $p$ in any model has the same value for the two components of the outer disk, that is, the disk surface and the midplane.

For $p \geq 0$, that is, when most of the mass is located in the outer regions of the disk, the PSF profile at $12.5 \mu \mathrm{m}$ is systematically resolved (red-box values in Table B.1). For a negative power law, we find that an overly small outer radius $R_{\mathrm{o}}$ does not produce a satisfactory fit of the SED. For large values of $R_{\mathrm{o}}$ (e.g., $150 \mathrm{au}$ ) and a negative power law, the flux in the far-IR and sub$\mathrm{mm}$ range tends to be underestimated. A best fit for the SED is found for $p=-1.5, R_{\mathrm{o}}=80 \mathrm{au}$, and $R_{\mathrm{i}}=10 \mathrm{au}$ with a nonreduced $\chi^{2}$ value of 302 (or $\chi_{r}^{2}=1.9$ for $N-v=157$ ). This model, for which the $12.5 \mu \mathrm{m}$ PSF is unresolved, is then chosen as the reference baseline model for characterizing the PSF profiles in the PAH filters.

We highlight that the approach adopted to isolate a disk model for further analysis holds some limitation: for the outer disk, other parameters may influence the result, such as, for example, the flaring index $\beta$ not included in the minimization 
process. Nevertheless, during the search for a reference baseline model, we examined the influence of the flaring index and found that as soon as $\beta$ reaches $2 / 7$, the infrared excess at $5 \mu \mathrm{m}$ is overestimated.

This effect cannot be satisfactorily compensated by a reduction of the disk's mass, which would then result in an emission deficit in the mid-IR and sub-mm ranges. The value of $\beta=1 / 7$ remains conservative in comparison to values in the literature. Figure 4 presents the resulting SED overplotted with the observational one, which show very good agreement over the whole spectral range.

\subsection{Analysis of the PSF profiles}

The best-model parametrized in Table 7 is used to produce synthetic images at $8.6 \mu \mathrm{m}, 11.3 \mu \mathrm{m}$, and $12.5 \mu \mathrm{m}$ that are then convolved with the GTC PSF, and for which profiles are compared to our observations. Figure 5 presents the result of the comparison in the three different filters. The size of each symbol corresponds to roughly the $3-\sigma$ error on the mean of each radial point of the PSF.

We observe experimentally that the observed science PSF profile (black empty circles) is spatially resolved with respect to the calibrator PSF (red empty squares) between $\sim 0.1$ and $0.3^{\prime \prime}$. This is also clearly repeatable when using the two nearby calibrator stars (see caption). This is observed in the two PAH filters and the continuous vertical lines correspond to the FWHM values established in Table 2 for the science and calibration targets. On the contrary, the observed profiles in the Si- 6 filter at $12.5 \mu \mathrm{m}$ do not show a detectable difference in their FWHM, suggesting that the disk's extended emission is not resolved by CanariCam in the thermal continuum. We note that this comparison for the Si-6 filter is made only with the PSF calibrator second in time, as the first one suffered from poorer observing conditions. We also observe that the difference in FWHM between the science and the calibrator PSFs is more prominent in the PAH-1 filter than in the PAH-2 filter.

In order to better understand the spatial properties of the thermal emission in different bands, we compare the synthetic PSF profiles derived from the best-fit model of Table 7 with our observations (blue dash-line). We clearly see that this model of thermal emission has the same profile as the PSF calibrator and is likely unresolved in our three observing bands. It is possible that another source of emission needs to be invoked to explain our observations.

\section{Discussion}

\subsection{Spatially resolved PAH circumstellar emission}

Our measurements show spatially resolved emission in the PAH-1 and PAH-2 filters centered on the infrared emission bands (IEBs) at 8.6 and $11.3 \mu \mathrm{m}$. These correspond to the two most prominent PAH bands in the $8-13 \mu \mathrm{m}$ region of the spectrum of HD 179218. The bulk of the emission detectable by our observations has a spatial extent of $\sim 12-15$ au in radius, assuming $d=293 \mathrm{pc}$. No disk emission is resolved in the $12.5 \mu \mathrm{m}$ filter, where the emission is dominated by the dust thermal continuum according to the spectrum (Juhász et al. 2010). Advantageously, the angular diameter of the derived Gaussian disk model of HD 179218 can be compared to other existing interferometric measurements using the same model. Our Gaussian FWHM of $95 \pm 6$ mas and $101 \pm 7$ mas in the PAH- 1 and PAH-2 filters, respectively, is larger than the Gaussian FWHM of $80 \pm 3$ mas at
$10.7 \mu \mathrm{m}(\Delta \lambda=1.45 \mu \mathrm{m})$ measured for the disk's thermal continuum by Monnier et al. (2009) using aperture masking. Nulling interferometry measurements by Liu et al. (2007) revealed a Gaussian FWHM of $81 \pm 16$ mas at $10.6 \mu \mathrm{m}$, although over a wide $50 \%$ bandpass encompassing the whole $\mathrm{N}$ band. Finally, our upper limit measured in the Si- 6 filter appears very coherent with the Gaussian FWHM of $0.034^{\prime \prime} \pm 3 \%$ estimated by Leinert et al. (2004) at $12.5 \mu \mathrm{m}$ using MIDI/VLTI. Previous singleaperture mid-infrared imaging observations did not resolve at $11.6 \mu \mathrm{m}(\Delta \lambda=1.1 \mu \mathrm{m}$; Mariñas et al. 2011) and in the $\mathrm{Q}$ band (Mariñas et al. 2011; Honda et al. 2015). These observations were however conducted with Gemini and Subaru, which deliver intrinsically poorer spatial resolution than the GTC by a factor $\sim 1.2$ and were not done in the PAH filters.

In a second step, our radiative transfer modeling helped to investigate the nature of the resolved emission. Fitting both the SED data and our imaging data with a model containing a passive disk suggests that the dust thermal emission alone would not be resolved by our CanariCam observations. We suggest that the detected resolved emission is caused by PAH molecules UVexcited by the central star and located near the surface of the flared disk. An interesting comparison can be advanced here with HD 97048, a notable Herbig star for which the PAH emission at 8.6 and $11.3 \mu \mathrm{m}$ is resolved out to several tens of astronomical units (Lagage et al. 2006). Doucet et al. (2007) estimated the disk diameter $D_{r m d}$ of HD 97048 - in the sense of the quadratic subtraction used above - to be $\sim 40$ au at a distance of $180 \mathrm{pc}$, which is slightly larger than for HD 179218. However, HD 97048 being about two times closer, the disk is better resolved in its outer regions. Despite a relatively narrow science PSF core (about 1.2 times larger than the PSF calibrator core), a large amount of PAH emission is found in the wings of HD 97048's PSF out to $380 \mathrm{au}$ in radius when comparing it with the emission in the immediate nearby continuum (SIV filter at $10.49 \mu \mathrm{m}$, see Fig. 3 in Doucet et al. 2007).

Similarly, in the case of HD 179218, the analysis presented in Sect. 4 may only provide the characteristic size of the disk's emission, but does not allow to constrain the true physical spatial extent of the PAH emission with respect to the continuum emission. For this purpose, it is necessary to account for the flux ratio between the PAH and continuum components in each filter. This can be estimated by subtracting from our photometric measurements in Table 4 the flux density of the PAH contribution at 8.6 and $11.3 \mu \mathrm{m}$ as estimated by Juhász et al. (2010, Table 9). We derive a continuum flux density of $11.4 \mathrm{Jy}$ and $19.1 \mathrm{Jy}$ for a corresponding PAH flux density of $3.9 \mathrm{Jy}$ and $2.9 \mathrm{Jy}$, respectively at 8.6 and $11.3 \mu \mathrm{m}$. We then build a hybrid model based on the image of the HD 179218's system in the continuum obtained by radiative transfer simulations (cf. Sect. 5) to which we superimpose a geometrical model simulating the PAH brightness distribution. The relative flux density of each component is scaled accordingly. For the PAH emission component, we investigated a Gaussian model and uniform disk (UD) modified model with radius $R_{\mathrm{UD}}$ modulated by a radial power law $r^{p}$. Using the former model, we are able to fit the core of the science PSF but we underestimate the emission in its wings in particular at $8.6 \mu \mathrm{m}$, whereas the smoother radial profile of the latter UD model better reproduces the full science PSF profile: as presented in Fig. 6, we find that in both filters a classical UD model with $p=0, R_{\mathrm{UD}}=0.3^{\prime \prime}(87 \mathrm{au}$ at $293 \mathrm{pc}$ ) and scaled to the corresponding PAHs flux density successfully matches the science PSF profiles of HD 179218. This result shows that, despite the small characteristic size - in the sense of quadratic subtraction - of HD 179218's disk emission, the PAHs emission at 8.6 and 

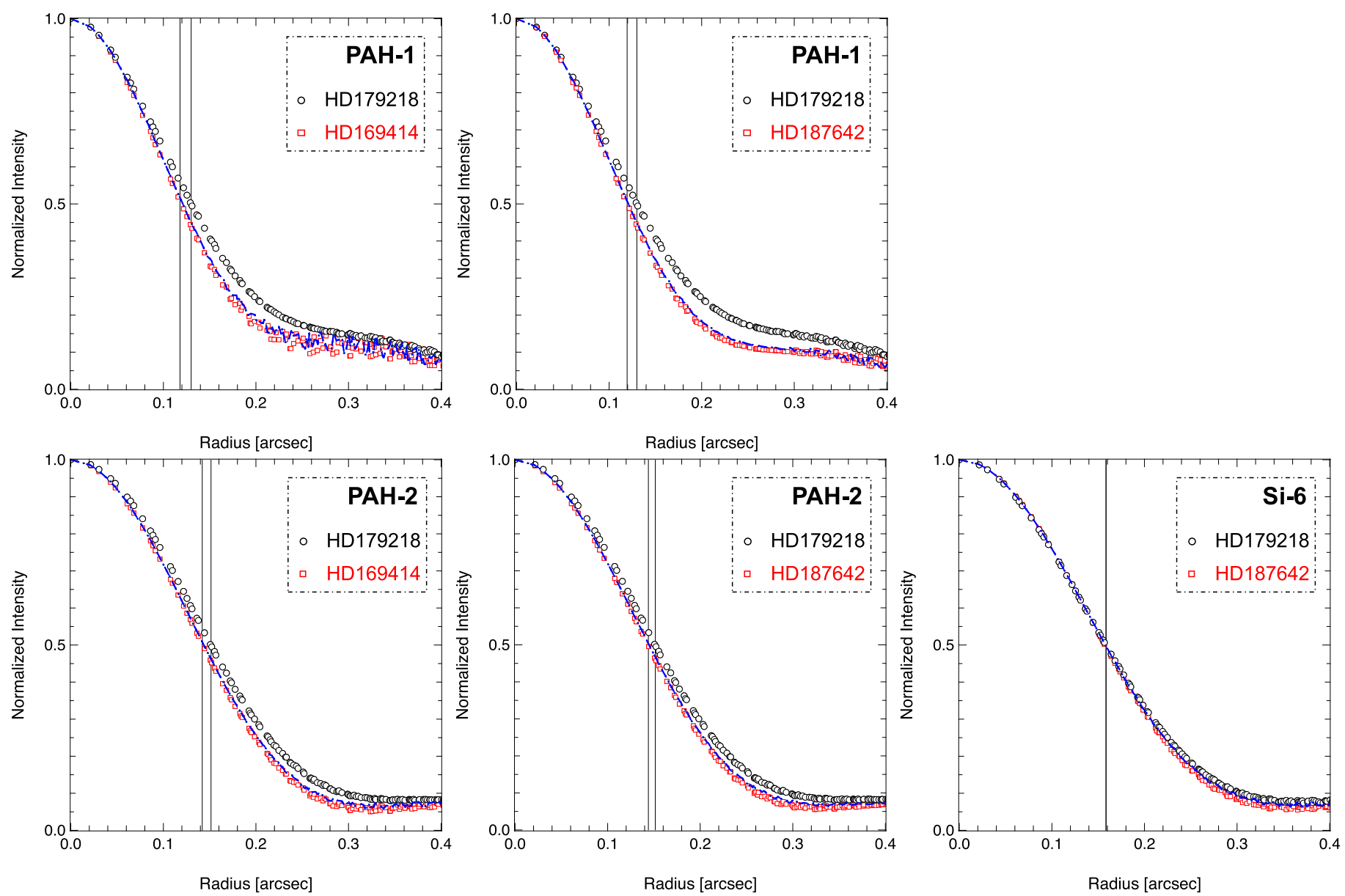

Fig. 5. Overview of the science, calibrator, and model PSF profiles. In all views, the open red squares refer to the calibrator sources and the open black circles correspond to the science target. For each filter, PAH-1 and PAH-2, the left plot corresponds to the comparison between the science and the first calibrator PSF profiles, whereas the right plot compares the science and the second calibrator PSF profiles. The size of the error bar for each point is similar to the size of the corresponding symbol. The dashed blue line is the radial profile obtained after convolution of the corresponding PSF calibrator with the radiative transfer model derived in Sect. 5. The vertical continuous lines show the difference in FWHM between the calibrator and the science for the values reported in Table 2.
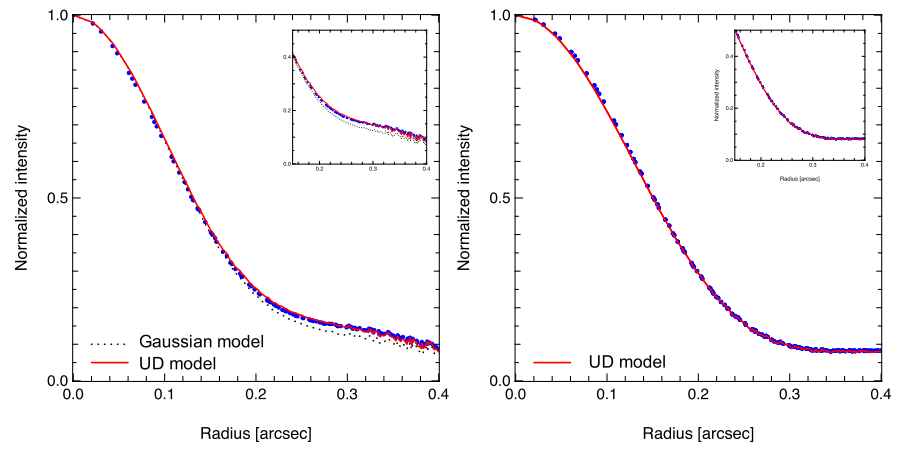

Fig. 6. Modeling of the science PSF profile (blue filled large dots) in the PAH-1 (left) and PAH-2 (right) filter with our hybrid model based on a Gaussian disk (black dotted line) and on the uniform disk (continuous red line) models, respectively. The insets show a close view on the wings of the PSF.

$11.3 \mu \mathrm{m}$ extends comparatively out to larger radii, which suggests a spatial scenario of the disk's emission similar to HD 97048.

\subsection{Charge state of PAHs in HD 179218}

Our results can be replaced in the larger context of the study of PAH emission in Herbig stars' disks. When looking at the question of PAHs spatial extent and charge state (ionized vs. neutral) in more detail, the recent studies by Maaskant et al. $(2013,2014)$ highlight more complex scenarios. It is found from four typical Herbig objects that the bulk of the PAH emission in (pre)transitional Herbig systems can originate either in the inner (optically thin) or the outer (optically thick) region of the protoplanetary disk. Observationally, this results in a PAH emission component with a smaller, comparable or larger characteristic size than the thermal continuum emission. Two representative cases of, respectively, compact and extended emission are seen in IRS 48 (Geers et al. 2007) and HD 97048 (Lagage et al. 2006). Interestingly, Maaskant et al. (2014) suggests a correlation between a) the relative spatial extent of the PAH emission with respect to the thermal continuum and b) the charge state of the PAH molecules as classically traced by diagnostics such as the $I_{6.2} / I_{11.3}$ or $I_{3.3} / I_{7.7}$ feature ratios - see Fig. 21 in Peeters et al. (2002) - or the relative strength of the $7.7+8.6 \mu \mathrm{m}$ feature compared to the $11.3 \mu \mathrm{m}$ feature. In the archetypical case of HD 97048, the PAH emission is found to be significantly more extended than the thermal continuum at both 8.6 and $11.3 \mu \mathrm{m}$ (Lagage et al. 2006; Doucet et al. 2007; Maaskant et al. 2013). At the same time, the object's mid-IR spectroscopy is indicative of an emission caused predominantly by neutral PAH molecules traced by the strong 3.3 and 11.3 features (Seok \& Li 2017; Maaskant et al. 2014). 

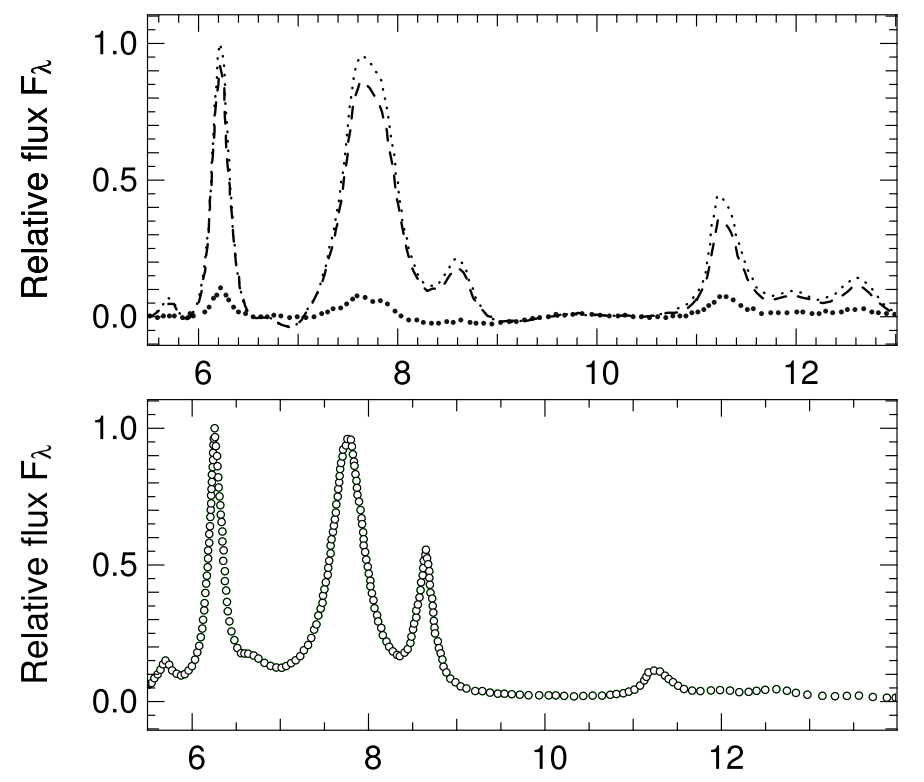

Wavelength $[\mu \mathrm{m}]$

Fig. 7. PAHs emission (i.e., continuum-subtracted) models for IRS 48 (top) and HD 179218 (bottom) adapted from Maaskant et al. (2014) and Seok \& Li (2017), respectively. The spectra are normalized to the peak emission at $6.3 \mu \mathrm{m}$. Top: the small-dotted line corresponds to the total PAH emission, the dashed line to the emission from ionized PAHs in optically thin environments like the gap, and the large-dotted line corresponds to the emission from neutral PAHs in the optically thick disk. Bottom: the model for the total PAH emission in HD 179218 shows a prominent feature at $8.6 \mu \mathrm{m}$ and a weaker feature at $11.3 \mu \mathrm{m}$.

The opposite case is found with IRS 48 where the ionized state of PAHs, as suggested by a $I_{6.2} / I_{11.3}$ ratio larger than unity, goes together with the $11.3 \mu \mathrm{m}$ PAH emission size being more compact than the continuum emission (Maaskant et al. 2014).

In HD 179218, different works based on ISO and Spitzer spectroscopy have reported and confirmed the relatively stronger $8.6 \mu \mathrm{m}$ PAH feature compared to the $11.3 \mu \mathrm{m}$ one (Meeus et al. 2001; Acke et al. 2010; Juhász et al. 2010; Seok \& Li 2017). Looking at the continuum-subtracted spectrum of HD 179218 modeled in Seok \& Li (2017) and qualitatively comparing it to the one of IRS 48 indicates similarities between the two spectra in terms of strength of the 6.2 and $7.7 \mu \mathrm{m}$ features compared to the $11.3 \mu \mathrm{m}$ feature (see Fig. 7). At first, this could point to a scenario for HD 179218 similar to IRS 48 with predominantly ionized PAHs located, for example, in the inner optically thin gap. However, our mid-IR imaging results and emission modeling suggest that the PAH contribution is not confined to the inner 10 au region of HD 179218, but may extend out to the outer disk regions, where a neutral charge state of the PAHs may be favored. A possible explanation to this scenario is that the stellar luminosity of HD $179218\left(L=180 L_{\odot}\right.$; Alecian et al. 2013) being significantly larger than for HD $97048\left(L \sim 40 L_{\odot}\right.$; Lagage et al. 2006; Maaskant et al. 2014), HD 169142 ( $L \sim 10 L_{\odot}$; Alecian et al. 2013; Maaskant et al. 2014) or for HD 135344 ( $L \sim 10$ $15 L_{\odot}$; Alecian et al. 2013; Maaskant et al. 2014), the central star consequently produces a stronger UV radiation field capable of ionizing PAH molecules out to larger distances. A plausible hypothesis could also be that PAH ionization out to large distances results from a wide-angle wind impinging on the disk surface. In a different context, this scenario is observed in highermass evolved Wolf-Rayet stars (Marchenko \& Moffat 2017). Ideally, the wind scenario could be investigated with IR interferometry by resolving the spatial size of the Br $\gamma$ emission line detected in HD 179218 (Garcia Lopez et al. 2006) and comparing it to the size of the nearby continuum, similarly to the case of MWC 297, whose $\mathrm{Br} \gamma$ emission is driven by a diskwind mechanism (Malbet et al. 2007). For example, in the case of HD 97048, recent GRAVITY interferometric observations revealed a $\mathrm{Br} \gamma$ emission more compact than the nearby continuum (K. Rousselet-Perraut, priv. comm.) possibly indicative of a magnetospheric accretion process mechanism taking place in the very inner disk region (Kraus et al. 2008). Lacking a clear disk-wind mechanism could hence justify the survival and abundance of neutral PAH in that system. It is particularly interesting to note that, by tracing the ro-vibrational line of molecular $\mathrm{H}_{2}$ at $2.12 \mu \mathrm{m}$ in HD 97048's disk, Bary et al. (2008) conclude at a quiescent state of $\mathrm{H}_{2}$ that is not shocked nor entrained in a fast-moving wind or outflow associated with this young source.

\subsection{An alternative disk radiative transfer model}

In this work, we also propose an alternative radiative transfer model to Dominik et al. (2003) for the disk of HD 179218 by fitting simultaneously the SED and our imaging data. Dust thermal emission and isotropic scattering are considered in our radiative transfer model, but PAH emission is not included. Keeping in mind the uncertainty on the object's parallax, our best model implies a larger outer disk than D03 with $R_{\mathrm{O}}=80$ au and, in particular, a negative power-law with $p=-1.5$ for the outer disk surface density. This is slightly lower than the $p=-1$ found for the outer disks of similar group Ia/b objects like HD 100546 (Tatulli et al. 2011), HD 139614 (Matter et al. 2016) or AB Aur (di Folco et al. 2009). This is nonetheless opposite to the positive power-law $(p=+2)$ derived by D03, and we find that any positive dust density law in which most of the mass is located in the outer regions of the disk would result in a surface brightness distribution that should be resolved by our observations at $12.5 \mu \mathrm{m}$.

\section{Conclusions}

We conducted mid-infrared imaging and spectroscopic observations of the Herbig star HD 179218 using CanariCam on the GTC and obtained the following results:

- Helped by good weather conditions and by the format of CanariCam images into cubes of short duration savesets, we were able to obtain close to diffraction limited images of HD 179218, and among the sharpest N-band images obtained from the ground with a FWHMs of $\sim 210$ mas at $8 \mu \mathrm{m}$. By re-centering and combining a large number of savesets, we reached $3 \sigma$ uncertainties of less than 5 mas on the FWHM. With this potential, we resolve for the first time the circumstellar emission around HD 179218 in the PAH bands at 8.6 and $11.3 \mu \mathrm{m}$ and found characteristic size of $\sim 100$ mas in diameter.

- We performed photometry of the system at 8.6, 11.3 and $12.5 \mu \mathrm{m}$ and found values consistent with published flux densities and without noticeable variability within the measurement errors. The CanariCam low-resolution spectrum matches quite well the shape measured by Spitzer, except in the region of the Earth's ozone band where the spectral calibration is found to be unreliable. 


\section{A. S. Taha et al.: Extent of PAHs emission in HD 179218}

- Importantly, the combination of our imaging data with radiative transfer modeling suggests that the spatially resolved emission at 8.6 and $11.3 \mu \mathrm{m}$ is not of thermal equilibrium nature but may originate from UV-excited PAH molecules located at the surface of the flared disk. By taking into account the relative flux ratios between the PAH and thermal component, we find that our observations are best reproduced with a model of PAH "disk" extending out to the physical limits of the dust disk model.

- We discuss the compatibility of such a spatial scenario with the spectroscopic evidence that a predominant fraction of the PAH molecules might be in an ionized charge state. We suggest that a particularly strong UV radiation field from the star or a disk wind may ionize the PAH molecules out to the largest radii.

- In contrast to the disk model already proposed by Dominik et al. (2003), our alternative radiative transfer model of HD 179218 coupled to mid-infrared imaging at $12.5 \mu \mathrm{m}$ suggests a surface density with a $p=-1.5$ negative power-law index, with most of the dust mass located in the first 30 au of the outer disk. Assuming that the gas and the PAH molecules are strongly coupled, the detection of an extended PAH emission would point to a flared structure of the disk in HD 179218 and confirm earlier results.

Acknowledgements. This work is fully supported by the University of Cologne, the Ministry of Higher Education and Scientific Research in Iraq (MoHERS), the Bonn-Cologne Graduate School of Physics and Astronomy (BCGS) and the University of Baghdad. We are thankful to all members of the Gran Telescopio Canarias (GTC) telescope for their support during the observations. We would also like to express our gratitude to the Sky Team of the IAC for providing us with the seeing data. We thank the anonymous referee for assessing the quality of this work.

\section{References}

Acke, B., \& van den Ancker, M. E. 2004, A\&A, 426, 151

Acke, B., Bouwman, J., Juhász, A., et al. 2010, ApJ, 718, 558

Alecian, E., Wade, G. A., Catala, C., et al. 2013, MNRAS, 429, 1027

Bary, J. S., Weintraub, D. A., Shukla, S. J., Leisenring, J. M., \& Kastner, J. H 2008, ApJ, 678, 1088

Benisty, M., Juhasz, A., Boccaletti, A., et al. 2015, A\&A, 578, L6
Bouwman, J., Meeus, G., de Koter, A., et al. 2001, A\&A, 375, 950 Calvet, N., D’Alessio, P., Hartmann, L., et al. 2002, ApJ, 568, 1008 di Folco, E., Dutrey, A., Chesneau, O., et al. 2009, A\&A, 500, 1065 Dominik, C., Dullemond, C. P., Waters, L. B. F. M., \& Walch, S. 2003, A\&A, 398,607

Doucet, C., Habart, E., Pantin, E., et al. 2007, A\&A, 470, 625

Dullemond, C. P., \& Dominik, C. 2004, A\&A, 417, 159

Espaillat, C., D’Alessio, P., Hernández, J., et al. 2010, ApJ, 717, 441

Fedele, D., van den Ancker, M. E., Acke, B., et al. 2008, A\&A, 491, 809

Furlan, E., Hartmann, L., Calvet, N., et al. 2006, ApJS, 165, 568

Garcia Lopez, R., Natta, A., Testi, L., \& Habart, E. 2006, A\&A, 459, 837

Geers, V. C., van Dishoeck, E. F., Visser, R., et al. 2007, A\&A, 476, 279

González-Martín, O., Rodríguez-Espinosa, J. M., Díaz-Santos, T., et al. 2013, A\&A, 553, A35

Gräfe, C., Wolf, S., Roccatagliata, V., Sauter, J., \& Ertel, S. 2011, A\&A, 533, A89

Habart, E., Boulanger, F., Verstraete, L., Walmsley, C. M., \& Pineau des Forêts, G. 2004, A\&A, 414, 531

Honda, M., Maaskant, K., Okamoto, Y. K., et al. 2012, ApJ, 752, 143

Honda, M., Maaskant, K., Okamoto, Y. K., et al. 2015, ApJ, 804, 143

Juhász, A., Bouwman, J., Henning, T., et al. 2010, ApJ, 721, 431

Kraus, S., Hofmann, K.-H., Benisty, M., et al. 2008, A\&A, 489, 1157

Lagage, P.-O., Doucet, C., Pantin, E., et al. 2006, Science, 314, 621

Leinert, C., van Boekel, R., Waters, L. B. F. M., et al. 2004, A\&A, 423, 537

Li, D. 2014, iDealCam: Interactive Data Reduction and Analysis for CanariCam (Astrophysics Source Code Library)

Liu, W. M., Hinz, P. M., Meyer, M. R., et al. 2007, ApJ, 658, 1164

Maaskant, K. M., Honda, M., Waters, L. B. F. M., et al. 2013, A\&A, 555, A64

Maaskant, K. M., Min, M., Waters, L. B. F. M., \& Tielens, A. G. G. M. 2014, A\&A, 563, A78

Malbet, F., Benisty, M., de Wit, W.-J., et al. 2007, A\&A, 464, 43

Marchenko, S. V., \& Moffat, A. F. J. 2017, MNRAS, 468, 2416

Mariñas, N., Telesco, C. M., Fisher, R. S., \& Packham, C. 2011, ApJ, 737, 57

Mathis, J. S., Rumpl, W., \& Nordsieck, K. H. 1977, ApJ, 217, 425

Matter, A., Labadie, L., Augereau, J. C., et al. 2016, A\&A, 586, A11

Meeus, G., Waters, L. B. F. M., Bouwman, J., et al. 2001, A\&A, 365, 476

Menu, J., van Boekel, R., Henning, T., et al. 2015, A\&A, 581, A107

Moerchen, M. M., Telesco, C. M., Packham, C., \& Kehoe, T. J. J. 2007, ApJ, 655, L109

Moerchen, M. M., Telesco, C. M., \& Packham, C. 2010, ApJ, 723, 1418

Monnier, J. D., Tuthill, P. G., Ireland, M., et al. 2009, ApJ, 700, 491

Peeters, E., Hony, S., Van Kerckhoven, C., et al. 2002, A\&A, 390, 1089

Seok, J. Y., \& Li, A. 2017, ApJ, 835, 291

Tatulli, E., Benisty, M., Ménard, F., et al. 2011, A\&A, 531, A1

Telesco, C. M., Ciardi, D., French, J., et al. 2003, in Proc. SPIE, 4841, eds. M. Iye, \& A. F. M. Moorwood, 913

Woitke, P., Min, M., Pinte, C., et al. 2016, A\&A, 586, A103

Wolf, S., Malbet, F., Alexander, R., et al. 2012, A\&ARv, 20, 52 


\section{Appendix A: Cumulative FWHM}

The following plots in Fig. A.1 illustrate how the sub-pixel re-centering of the individual savesets with respect to each other allows us to minimize the broadening of the PSF that could result from long integrations or basic stacking.
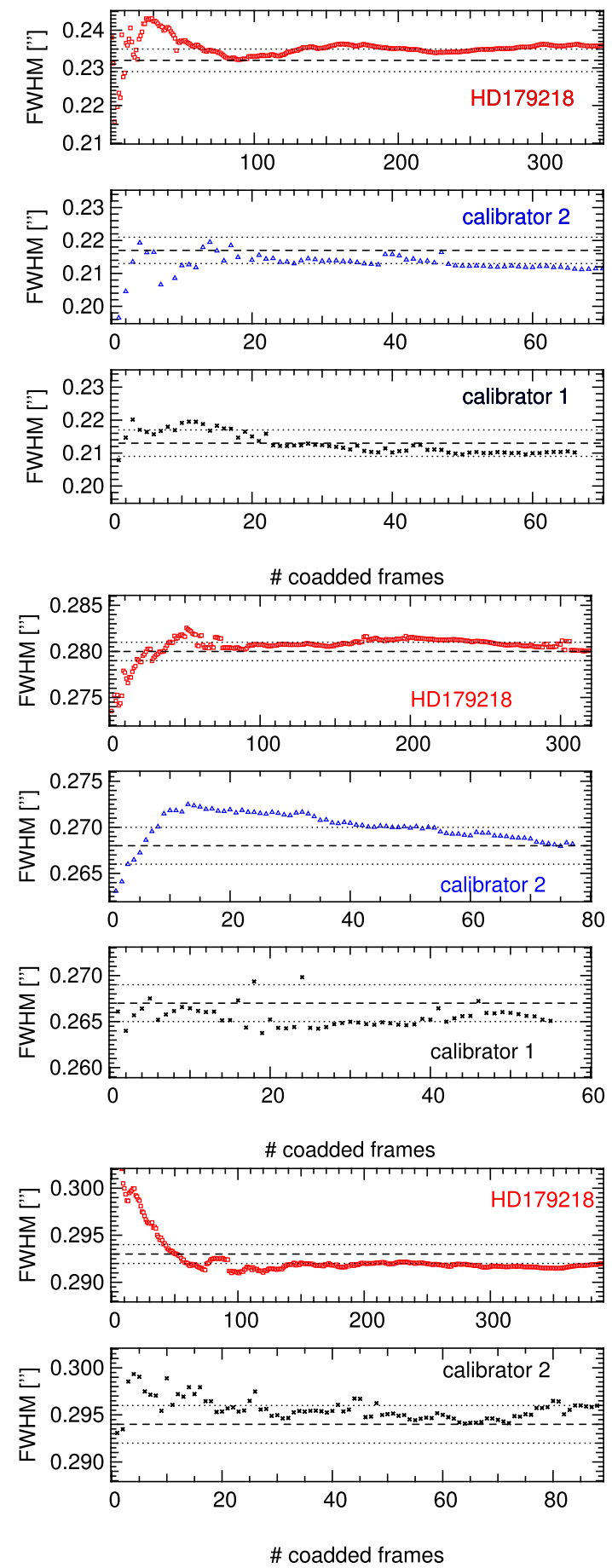

Fig. A.1. Cumulative FWHM for both reference stars (HD 169414 and HD 187642) and science star (HD 179218) in the PAH-1 (top), PAH2 (center) and Si-6 (bottom) filters as a function of the number of co-added frames following the procedure described in Sect. 3.2. The dashed line is the average FWHM reported in Table 2 and following a Lorentzian fit, whereas the dotted lines correspond to the $3 \sigma$ boundaries.

\section{Appendix B: Results of $\chi^{2}$ minimization}

Table B.1 reports the value of the non-reduced $\chi^{2}$ on the SED obtained during the search of our best radiative transfer model of the disk's thermal emission in HD 179218. The observational SED is plotted in Fig. 4. We compare a posteriori the synthetic and the observed PSF profiles at $12.5 \mu \mathrm{m}$.

Table B.1. Non-reduced $\chi^{2}$ table for the fit of the SED for different values of $p$ in Eq. (3), $R_{\mathrm{i}}$ and $R_{\mathrm{o}}$, respectively the inner and outer radius of the outer disk in HD 179218.

\begin{tabular}{|c|c|c|c|c|c|}
\hline \multicolumn{6}{|c|}{$R_{\mathrm{i}}$} \\
\hline & $8 \mathrm{au}$ & $9 \mathrm{au}$ & $10 \mathrm{au}$ & $11 \mathrm{au}$ & $12 \mathrm{au}$ \\
\hline$p=-2, R_{\mathrm{o}}=30 \mathrm{au}$ & 3557 & 2947 & 2433 & 2285 & 1935 \\
\hline$p=-1.5, R_{\mathrm{o}}=30 \mathrm{au}$ & 3044 & 2557 & 2206 & 2076 & 1814 \\
\hline$p=-1, R_{\mathrm{o}}=30 \mathrm{au}$ & 2553 & 2253 & 1944 & 1912 & 1623 \\
\hline$p=0, R_{\mathrm{o}}=30 \mathrm{au}$ & 1967 & 1731 & 1690 & 1665 & 1505 \\
\hline$p=+1, R_{\mathrm{o}}=30 \mathrm{au}$ & 1781 & 1676 & 1559 & 1547 & 1418 \\
\hline$p=+2, R_{\mathrm{o}}=30 \mathrm{au}$ & 1823 & 1669 & 1637 & 1621 & 1478 \\
\hline$p=-2, R_{\mathrm{o}}=50 \mathrm{au}$ & 1493 & 1094 & 850 & 718 & 585 \\
\hline$p=-1.5, R_{\mathrm{o}}=50 \mathrm{au}$ & 974 & 758 & 607 & 547 & 454 \\
\hline$p=-1, R_{\mathrm{o}}=50 \mathrm{au}$ & 578 & 482 & 448 & 443 & 408 \\
\hline$p=0, R_{\mathrm{o}}=50 \mathrm{au}$ & 469 & 500 & 529 & 534 & 566 \\
\hline$p=+1, R_{\mathrm{o}}=50 \mathrm{au}$ & 848 & 840 & 848 & 853 & 841 \\
\hline$p=+2, R_{\mathrm{o}}=50 \mathrm{au}$ & 1132 & 1124 & 1114 & 1112 & 1082 \\
\hline$p=-2, R_{\mathrm{o}}=80 \mathrm{au}$ & 727 & 503 & 376 & 309 & 291 \\
\hline$p=-1.5, R_{\mathrm{o}}=80 \mathrm{au}$ & 391 & 330 & 302 & 325 & 365 \\
\hline$p=-1, R_{\mathrm{o}}=80 \mathrm{au}$ & 346 & 407 & 443 & 530 & 609 \\
\hline$p=0, R_{\mathrm{o}}=80 \mathrm{au}$ & 1075 & 1154 & 1172 & 1227 & 1264 \\
\hline$p=+1, R_{\mathrm{o}}=80 \mathrm{au}$ & 1730 & 1707 & 1737 & 1743 & 1753 \\
\hline$p=+2, R_{\mathrm{o}}=80 \mathrm{au}$ & 1893 & 1890 & 1906 & 1898 & 1886 \\
\hline$p=-2, R_{\mathrm{o}}=100 \mathrm{au}$ & 633 & 471 & 373 & 330 & 333 \\
\hline$p=-1.5, R_{\mathrm{o}}=100 \mathrm{au}$ & 423 & 385 & 365 & 409 & 474 \\
\hline$p=-1, R_{\mathrm{o}}=100 \mathrm{au}$ & 477 & 565 & 610 & 682 & 781 \\
\hline$p=0, R_{\mathrm{o}}=100 \mathrm{au}$ & 1489 & 1489 & 1489 & 1489 & 1489 \\
\hline$p=+1, R_{\mathrm{O}}=100 \mathrm{au}$ & 2074 & 2097 & 2106 & 2112 & 2138 \\
\hline$p=+2, R_{\mathrm{o}}=100 \mathrm{au}$ & 2328 & 2330 & 2330 & 2360 & 2350 \\
\hline$p=-2, R_{\mathrm{o}}=150 \mathrm{au}$ & 644 & 548 & 499 & 449 & 550 \\
\hline$p=-1.5, R_{\mathrm{o}}=150 \mathrm{au}$ & 582 & 603 & 643 & 721 & 806 \\
\hline$p=-1, R_{\mathrm{o}}=150 \mathrm{au}$ & 777 & 898 & 987 & 1074 & 1226 \\
\hline$p=0, R_{\mathrm{o}}=150 \mathrm{au}$ & 1951 & 2012 & 2131 & 2173 & 2242 \\
\hline$p=+1, R_{\mathrm{o}}=150 \mathrm{au}$ & 2992 & 2965 & 2973 & 3015 & 3063 \\
\hline$p=+2, R_{\mathrm{o}}=150 \mathrm{au}$ & 3356 & 3385 & 3407 & 3360 & 3412 \\
\hline
\end{tabular}

Notes. The light and dark gray boxes correspond to models for which the $12.5 \mu \mathrm{m}$ PSF is spatially unresolved and resolved, respectively. The best model is identified for the value $\chi^{2}=302$ (bold). 